\title{
Tolerancia al material de osteosíntesis en cirugía electiva del maxilar superior
}

\author{
C. Alasà, J. González-Lagunas, E. Ariza, D. Malet, G. Raspall
}

\begin{abstract}
Resumen: Se evaluaron retrospectivamente un total de 36 pacientes sometidos a osteotomía de LeFort I. En 21 de estos pacientes ( 8 hombres y 13 mujeres con edad media 20,8 años) se realizaron osteotomías estándar con corrección del defecto con miniplacas en forma de «», usando en 11 casos miniplacas 2,0 y el resto 1,5. Se sometieron a los 15 pacientes restantes (8 hombres y 7 mujeres con edad media 39,6 años) a osteotomía segmentada sagital para el abordaje lesiones de la base del cráneo, colocando en todos ellos miniplacas de 2,0. Después de un seguimiento mínimo de 30 meses se llegaron a los siguientes resultados: De un total de 160 miniplacas colocadas ( 84 en grupo estándar y 76 en osteotomías de abordaje neuroquirúrgico) se retiraron sólo 4 de estas miniplacas, 2 de ellas por palpación intraoral y 2 por exposición. Por todo lo expuesto y en comparación con otras series concluimos que la baja incidencia de complicaciones derivadas del material de osteosíntesis no justifica su retirada sistemática en las osteotomías de maxilar superior.
\end{abstract}

Palabras clave: Osteotomía de LeFort; Miniplaca; Fijación rígida; Osteosíntesis.

\begin{abstract}
In Europe, in the late seventies, Lurh, Spiessel, Schilli and Champy, among others developed and popularized internal fixation with plates and screws with the aim of avoiding the need of maxillomandibular fixation in the treatment of maxillofacial injuries and deformities. In this paper the need to remove this materials after the consolidation time is discussed. There are some adverse circumstances that oblige the surgeon to remove the hardware like, palpation, exposure, migration, locally infection or thermal intolerance. This paper presents a retrospective study of thirty-six patients who underwent Le Fort osteotomy at the Department of Oral and Maxillofacial Surgery in Vall d'Hebron Hospital. Also, a review of the complications derived from the hardware material is presented. After a minimum follow-up of 30 months the following results were observed: only 4 of the 160 installed miniplates had to be removed. The low incidence of complications does not justify the systematic removal of ostheosynthesis material in this group of patients.
\end{abstract}

Key words: Le Fort osteotomy; Miniplate; Rigid fixation; Osteosynthesis.

\section{Introducción}

En Europa, a finales de la década de los 70, Lurh, ${ }^{1}$ Spiessel, ${ }^{2}$ Schilli, ${ }^{3}$ y Champy, ${ }^{4}$ desarrollaron y populari-

Servicio de Cirugía Oral y Maxilofacial.

Hospital Universitario Vall d'Hebron, Barcelona, España

Correspondencia:

Dr Javier González Lagunas

Casanova 101, Principal

08011 Barcelona, España

glagunas@terra.es zaron la fijación interna mediante miniplacas y tornillos con el objetivo de eliminar la necesidad del bloqueo intermaxilar en el tratamiento de las deformidades y fracturas máxilofaciales. La necesidad de retirar dichos materiales de osteosíntesis una vez transcurrido el periodo de consolidación es una controversia aun hoy en día. ${ }^{5}$ Existen diversas situaciones que obligan a retirar el material de osteosíntesis, tales como la palpación, la exposición, la migración, la infección local, o la sensibilidad al frío o al calor. ${ }^{5}$ En el presente artículo se presentan los resultados de un estudio retrospectivo de 36 pacientes sometidos a osteotomías maxilares de LeFort 


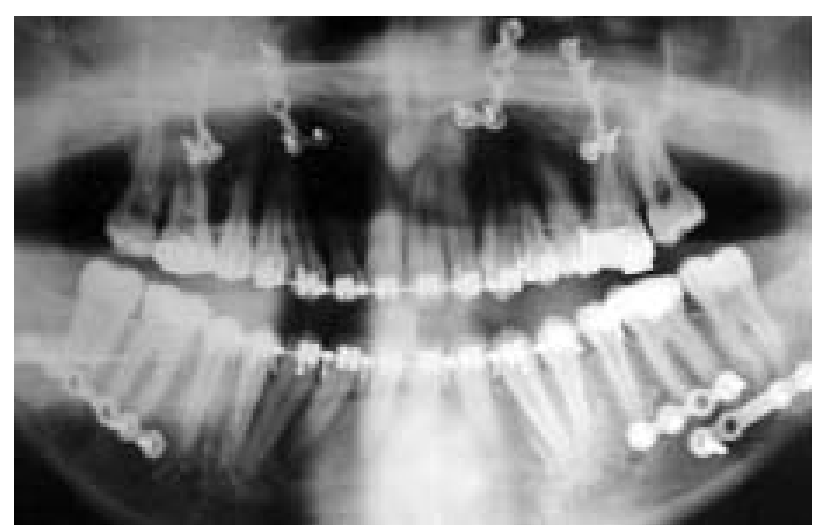

Figura 1. Ortopantomografía correspondiente a osteosíntesis en paciente afecto de deformidad dentofacial.

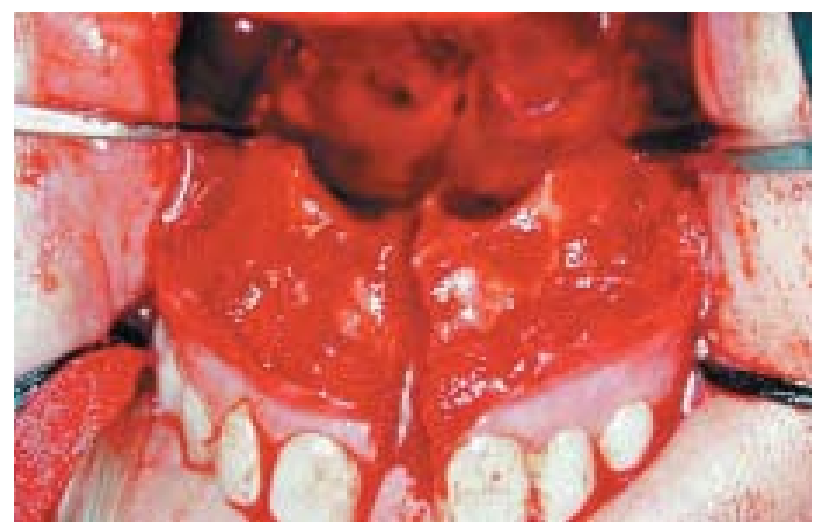

Figura 3. Maxilotomía para abordaje de base de cráneo.

I en el Departamento de Cirugía Oral y Maxilofacial del Hospital Vall d'Hebron, donde se evalúan las complicaciones derivadas del material de osteosíntesis.

\section{Material y método}

Se presenta un estudio retrospectivo sobre 36 pacientes sometidos a osteotomía de LeFort I, realizadas en el Servicio de Cirugía Oral y Maxilofacial del Hospital Universitario Vall d'Hebron, entre Enero de 1996 y Julio de 1997.

Veintiún pacientes $(58,3 \%)$ fueron sometidos a osteotomía de LeFort I estándar en el contexto de una cirugía ortognática. Estos pacientes fueron intervenidos quirúrgicamente bajo anestesia general. Se realizó una incisión vestibular superior que se extendía de primer molar superior derecho a primer molar superior izquierdo. Se practicaron las osteotomías con una sierra oscilante en la parte anterior y lateral del maxilar superior y una disyunción pterigomaxilar con esclopo inmediatamente por detrás del segundo molar superior. Una vez corregido el defecto, se fijó con miniplacas de titanio, una miniplaca en forma de $₫ \gg$ a cada lado de la escotadura piri-

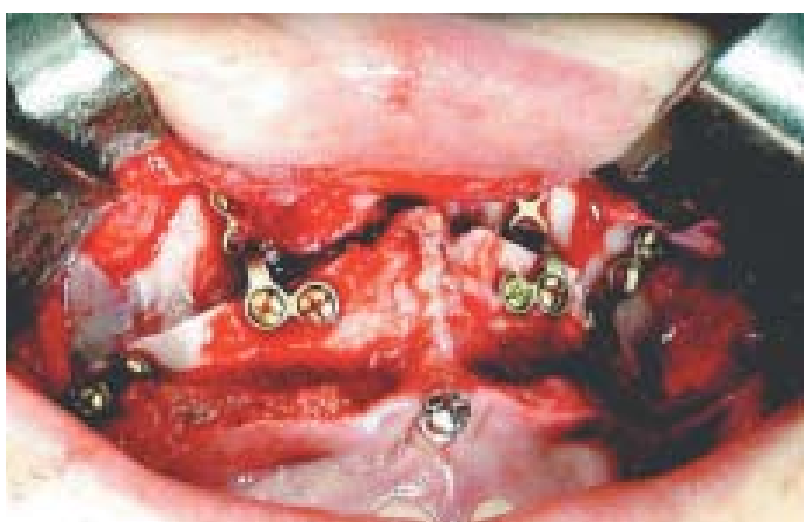

Figura 2. Imágen clínica donde se observa la posición de 4 miniplacas en maxilar superior.

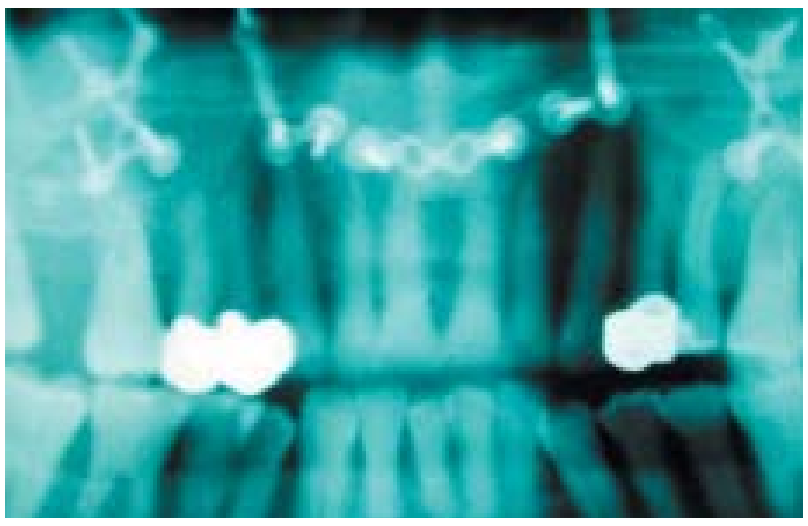

Figura 4. Fijación de la osteotomía de acceso con 5 miniplacas.

forme, y una miniplaca en forma de « $₫$ en cada uno de los arbotantes máxilo-malares (Figs. 1 y 2). En 11 pacientes se colocaron miniplacas de 2,0 y en 10 pacientes miniplacas de 1,5 .

El resto del grupo, lo forman 15 pacientes (41,6\%) sometidos a osteotomía segmentada sagital de LeFort I para el abordaje de lesiones de la base del cráneo. ${ }^{6}$ Estos pacientes fueron intervenidos bajo anestesia general. Se practicó abordaje intraoral transmucoso con una incisión vestibular que se extendía de segundo molar superior derecho a segundo molar superior izquierdo. Se practicó la osteotomía horizontal del maxilar con una sierra oscilante, la osteotomía medial interincisal se practicó también con la sierra oscilante realizando previamente un abordaje transmucoso en línea media del paladar. Una vez realizado el tratamiento neuroquirúrgico se fijó con 5 miniplacas de titanio, una miniplacas en forma de $\mathbb{L} \gg$ a cada lado de la escotadura piriforme, una miniplaca en forma de «X»en cada uno de los arbotantes máxilo-malares y una miniplaca recta en línea media maxilar (Figs. 3 y 4). En todos los pacientes de este grupo se colocaron miniplacas de 2,0.

La edad media en el grupo de osteotomías estándar fue de 20,8 años (rango de 19-27), con una distribución de 8 varones y 13 mujeres. 
En el grupo de osteotomías segmentadas de abordaje la edad media fue de 39,6 años (rango de 25-61). La indicación en dichos pacientes fue el abordaje a la base del cráneo para la corrección de malformaciones complejas de la chamela occipital, en nueve casos, en tres de ellos por cordomas cervicales y en los seis restantes por artritis reumatoide. De los 15 pacientes de este grupo 8 eran varones y 7 mujeres.

Se realizó un seguimiento mínimo en todos los pacientes de 30 meses, revisando las complicaciones derivadas del material de osteosíntesis así cómo los casos en que se realizó retirada de las miniplacas.

\section{Resultados}

Se colocaron un total de 159 miniplacas, 84 en el grupo de osteotomías por deformidades dentofaciales y 75 en el grupo de maxilotomías de abordaje neuroquirúrgico. De las 159 miniplacas instaladas, se retiraron cuatro (2,5\%). Dos se retiraron debido a la palpación intraoral que producía disconfort a los pacientes y las otras dos por exposición intraoral de parte o toda la miniplaca.

En el grupo de osteotomías estándar sólo se retiró una de las 84 miniplacas (1,19\%). Se trató de una placa de osteosíntesis paranasal que se palpaba de forma objetiva en la cavidad oral y cuya retirada solicitó el propio paciente.

Las tres restantes miniplacas retiradas pertenecían al grupo de pacientes con maxilotomías de abordaje, representando el $4 \%$ del total de miniplacas utilizadas en dicho grupo. Una de ellas se retiró por palpación intraoral y las dos restantes por exposición también intraoral.

El periodo medio de seguimiento de los pacientes previamente a la retirada del material de osteosíntesis fue de 13 meses.

En el periodo de seguimiento no se objetivaron casos de infección de la herida quirúrgica, dolor, alteración de la sensibilidad ni migración del material de osteosíntesis.

Dos pacientes varones, ambos con miniplacas de 2,0, y pertenecientes al grupo de osteotomías segmentadas de abordaje a base de cráneo, presentaron durante el primer mes de seguimiento signos clínicos de sinusitis maxilar que cedieron con el tratamiento médico, sin precisar hasta el momento del final del estudio la necesidad de retirada del material de osteosíntesis.

\section{Discusión}

La necesidad de la retirada del material de osteosíntesis en el campo de la cirugía maxilofacial es controvertida. Inicialmente los materiales de osteosíntesis se diseñaron para ser retirados de forma generalizada. Actualmente los materiales se diseñan para perma- necer en el organismo o bien para ser reabsorvidos por éste de forma paulatina. ${ }^{7}$

Existen diversos motivos por los cuales no se realiza una retirada sistemática del material de osteosíntesis. La oposición del propio paciente a una segunda cirugía cuando no existe un condicionante clínico es un factor determinante. Otro motivo es la pérdida de seguimiento de los pacientes sometidos a osteosíntesis. Existen además motivos económicos, puesto que supone un gasto añadido a un tratamiento quirúrgico inicial.

La baja incidencia de complicaciones derivadas del material de osteosíntesis, 2,5\% en nuestra serie, no justifica desde nuestro punto de vista la necesidad de la retirada sistemática de tornillos ni miniplacas de osteosíntesis en las osteotomías de maxilar superior. Otras series publicadas en la literatura presentan porcentajes también bajos de retirada del material de osteosíntesis. Alpert y cols., ${ }^{8}$ presentan una incidencia de retirada del material de osteosíntesis del $11 \%$, en una serie de 190 pacientes sometidos a osteotomía de LeFort I. De ellos en el 10,6\% existió alguna complicación derivada de las placas y tornillos que indicaron su retirada, siendo la causa más frecuente, en esta serie, la exposición intraoral.

Berryhill y cols. ${ }^{9}$ en una serie de 121 casos de pacientes pediátricos describe una incidencia de complicaciones derivadas del material de osteosíntesis maxilofacial del $22 \%$ con la necesidad de retirada del material en el 23\% de los pacientes.

Las complicaciones derivadas del material de osteosíntesis presentan una mayor incidencia en la patología traumática maxilofacial, ${ }^{10}$ con riesgo de contaminación. En estos casos existen lesiones de tejidos circundantes como las paredes mucosas de los senos, los sistemas de drenaje sinusales o heridas cutáneas contaminadas. Dichos factores predisponen a la presentación de complicaciones tales como la infección, la exposición y la palpación en determinadas áreas anatómicas como los rebordes orbitarios. ${ }^{11}$

Las aleaciones de titanio puro de los actuales materiales de osteosíntesis han minimizado la presencia de reacciones de toxicidad o alergia existentes en anteriores materiales de aleaciones de níquel, cromo o cobalto. ${ }^{12}$ Sí parece existir evidencias en la fijación y depósito de titanio en diferentes tejidos del organis-

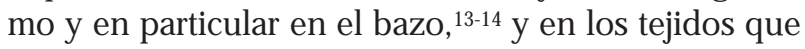
rodean los materiales de osteosíntesis que presentan déposito y reaccion local previo a la aparición de complicaciones. ${ }^{15}$

Dado que el material de osteosíntesis actúa como un cuerpo extraño favorecedor de la colonización de gérmenes, interesa ser lo más asépticos posibles durante el tratamiento quirúrgico, remarcando aspectos como la imigación intensa y continuada del lecho operatorio durante toda la intervención con la finalidad de disminuir al máximo la concentración de gérmenes. ${ }^{16}$ 
La irrigación profusa evita también la necrosis ósea que puede comprometer la viabilidad del material de osteosíntesis. La utilización de soluciones antisépticas previas al cierre de las incisiones operatorias y el cierre meticuloso y hermético de la mucosa oral suponen otra medida preventiva de importancia para la prevención de las infecciones. ${ }^{17}$

La mayor incidencia de complicaciones en el grupo de osteotomías segmentadas de abordaje posiblemente se explica tanto por la mayor agresión quirúrgica de la técnica operatoria como por la mayor duración de las intervenciones.

\section{Conclusiones}

Por todo lo expuesto y analizado en nuestro estudio y en comparación con otros estudios y los distintos artículos revisados, podemos concluir que existe un bajo número de complicaciones en la cirugía electiva del maxilar superior que, unido a la morbilidad y el coste económico de un segundo acto quirúrgico, no justifican la retirada sistemática del material de osteosíntesis. También cuesta justificar la necesidad de uso de materiales reabsorbibles en la cirugía del tercio medio facial, si se argumenta exclsuivamente con las complicaciones a largo plazo del material de titanio

\section{Bibliografía}

1. Lurh HG. Basic research, surgical technique and results of fracture treatment with the Luhr-Mandibular-Compression-Screw System. Proceedings from the Eighth International Conference on Oral and Maxillofacial Surgery, Berlin, Germany, Quintessence 1985:124.

2. Spiessel B. Internal Fixation of the Mandible. Manual of the AO/ASIF Principles. Berlin, Germany, Springer-Verlag 1989: pag.30-45.

3. Schilli W. Rigid internal fixation by means of compression plates. En Kruger E, Schilli W (eds). Oral and Maxillofacial Traumatology. Berlin, Quintessence 1982; pag.1.

4. Champy M, Loddge JP, Schmith R, Jaeger JH, Muster D. Mandibular osteosynthesis by miniature screwed plates via a buccal approach. J Oral Maxillofac Surg 1978;6:14-21.
5. Haug RH, y cols. Retention of asyntomatic bone plates used for orthognathic surgery and facial fractures. J Oral Maxillofacial Surg 1996;54:611-7.

6. Malet D. Base del craneo. En: Raspall G (ed). Cirugía Maxilofacial. Barcelona. Ed panamericana 1997: pag.41938.

7. Haers PE, Suuronen R, Lindqvist C, Sailer H. Biodegradable polylactide plates and screws in ortognathic surgery. J Craniomaxillofac Surg 1988;26:87-91.

8. Alpert B, Seligson D. Removal of asymptomatic bone plates used for orthognatic surgery and facial fractures. J Oral Maxillofac Surg 1996;54:618-21.

9. Berryhill WE, Rimel FL, Ness J, Marenttete L, Haines SJ. Fate of rigid fixation in pediatric craniofacial surgery. Otolaryngol-Head Neck Surg 1999;121: 26973.

10. Manor Y, Chaushu G, Taicher S. Risk factors contributing to symtomatic plate removal in orthognatic surgery patients. J Oral Maxillofacial Surg 1999;57:67982.

11. Brown JS, Trotter M, Cliffe J, Ward-Booth RP, Williams ED. The fate of miniplates in facial trauma and orthognatic surgery: a retrospective study. Br J Oral Maxillofacial Surg 1989;27:306-15.

12. Matthew I, Frame JW. Detection of titanium in human tissues after craniofacial surgery. Plast Reconstr Surg 1998;101:1148-9.

13. Katou F, Andoh N, Motegi K, Nagura H. Immuno-inflamatory responses in the tissue adjacent to titanium miniplates used in the treatment of mandibular fractures. J Craniomaxillofacial Surg 1996;24:155-62.

14. Woodman JL, Jacobs JJ, Galante JO, Urban RM. Metal ion release from titanium-based prosthetic segmental replacements of long bones in baboons. A long-term study. J Orthop Res 1984;1:421-30.

15. Acero J, Calderon J, Salmerón JI, Verdaguer JJ, Concejo $\mathrm{C}$, Somacarrera M. The behaviour of titanium as a biomaterial: microscopy study of plates and surrounding tissues in facial osteosynthesis. J Craneomaxillofac Surg 1999; 27:117-23.

16. Francel TJ, Birley BC, Ringelman PR, Manson PN. The fate of plates and screws after facial fracture reconstruction. Plast Reconstr Surg 1992;90:568-73.

17. Beals SP, Munro IR. The use of miniplates in craniomaxillofacial surgery. Plast Reconstr Surg 1987; 79:33-8. 